\title{
ULNAR NEURITIS
}

By Geoffrey Osborne, M.Ch.OrTh., F.R.C.S.E.

Orthopaedic Surgeon, Liverpool Royal Infirmary

Since the original description by Panas (1878), and the later comprehensive account by Platt (I926), the term ' ulnar neuritis' has been used to describe a progressive lesion of the ulnar nerve associated with deformity or abnormality of the elbow joint. Either osteo-arthritis, involving the ulnar groove, or cubitus valgus deformity, resulting from imperfect reduction of a childhood separation of the capitellar epiphysis, have been accepted as the common primary causes of a friction or traction neuritis, the nerve being constantly irritated during flexion movement of the elbow or stretched around a gradually increasing valgus angle. The term 'traumatic' ulnar neuritis was used to imply such frictional damage to the nerve or the effect of local injury due to posture or occupation. It was applied also to certain cases of ulnar palsy developing after pressure or injury to the hand (Russell and Whitty, 1947).

It was known, however, that a variety of ulnar nerve lesions can arise spontaneously (Ramsay Hunt, I9II), without any obvious injury to the nerve, and without any apparent abnormality of the elbow or other joints. The neuritis can occur at the level of the elbow, wrist or hand and, as the palsy is often incomplete, to establish the site of the lesion may prove a difficult clinical problem.

In the last few years the diagnosis of some of these less obvious cases of ulnar neuritis and ulnar palsy has been facilitated by the following recent work.

I. In 1952, Seddon described the occurrence of neuritis of the deep palmar branch of the ulnar nerve due to the compression of a ganglion arising from the joint between the triquetral and the hamate at the base of the hypothenar eminence (Fig. 2). Extensive paralysis of the interossei was present with sparing of the hypothenar muscles and sensory changes were absent. The condition occurred in middle age and was often associated with some initial or repeated trauma. The ganglion may not be clinically visible. Removal of the ganglion relieved the nerve compression but recovery could occur spontaneously, apparently due to gradual shrinkage of the ganglion. In the same

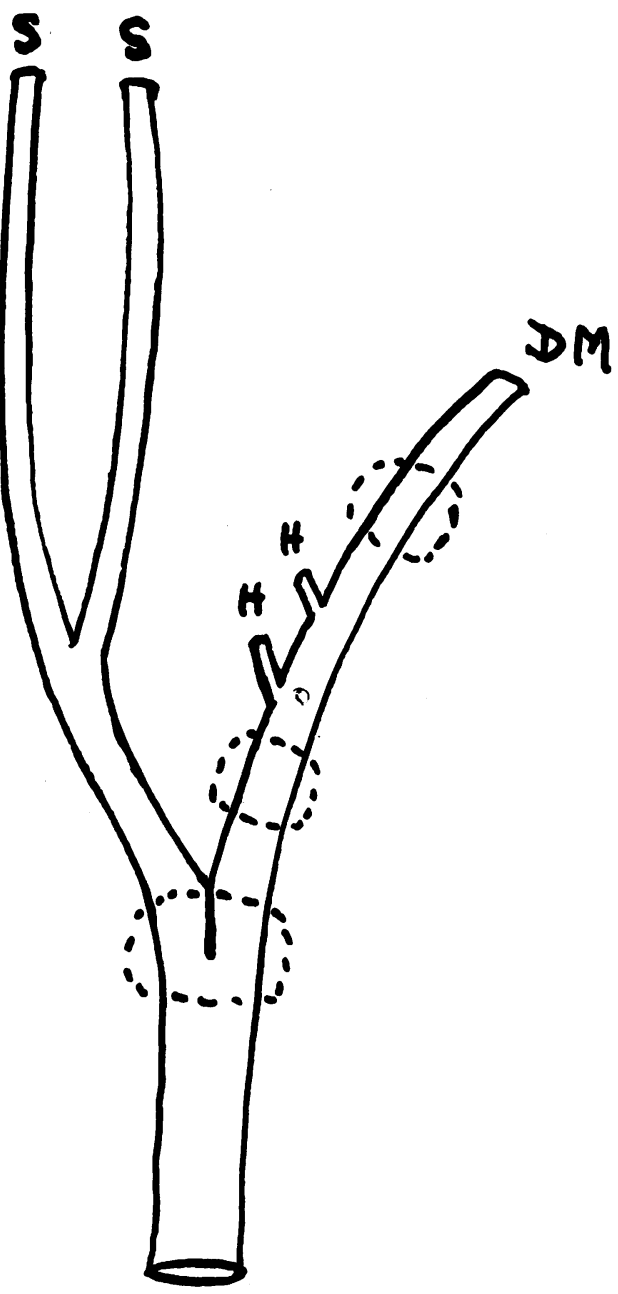

FIG. 1.-Diagram of distribution of ulnar nerve in palm, showing sites at which ganglia can cause $\omega$ compression.

$\mathrm{SS}=$ sensory branches.

$\mathrm{HH}=$ branches to hypothenar muscles.

$\mathrm{DM}=$ termination of deep motor branch interossei, lumbricals and adductor pollicis.

journal, Brooks also described ganglia arising more proximally from the wrist joint and compressing 


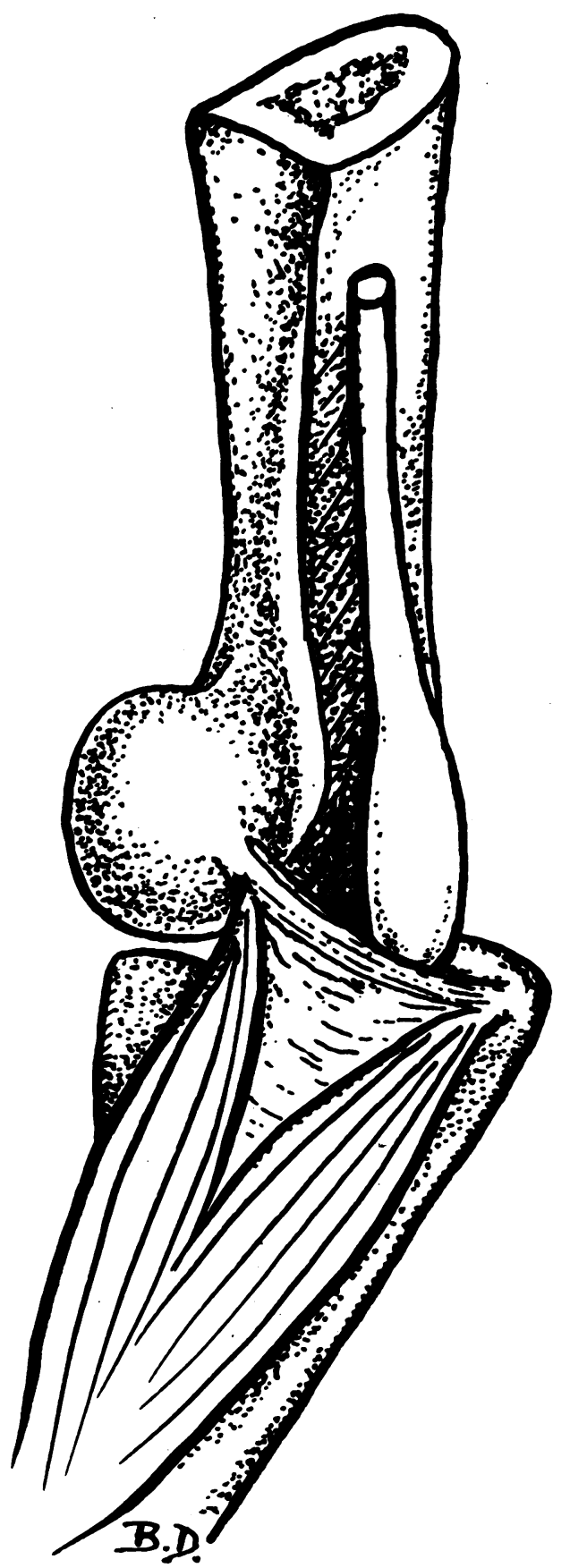

Fig. 2.-Fibrous-band bridging attachment of two heads of flexor carpi ulnaris, showing compression and proximal enlargement of nerve.

the whole ulnar nerve, or the motor branch with sparing of the sensory fibres to the ring and little fingers (Fig. I). Rarely, a fascial band attached to the pisiform can also cause ulnar nerve compres- sion. Ganglia arising at the elbow level from the trochleo-olecranon joint or the flexor carpi ulnaris may also affect the nerve. These ganglia are often small and arising from deep structures can be easily overlooked on examination. In the absence of an obvious swelling the features of nerve compression are not specific, and may not indicate the site of the lesion.

2. In 1957, Osborne described the results of a study of 25 cases of ulnar neuritis at the elbow, treated during the previous eight years.

He showed that whatever the underlying pathological changes in the elbow joint, the lesion affecting the nerve was identical in almost every case and consisted of compression of the nerve against a band of fibrous tissue bridging the two heads of flexor carpi ulnaris (Fig. 2). This band lay superficial to the nerve and constituted a definite condensation of transversely arranged fibres in the base of the aponeurosis, filling the triangular gap between the two heads of the muscle. It had received brief mention in text books as the 'tendinous arch ' or the ' arcuate ligament.' The band has a fixed attachment to the medial epicondyle and has a mobile attachment to the olecranon and is slack in full extension, but as the elbow is flexed it starts to tighten at $135^{\circ}$ and at $90^{\circ}$ it becomes quite taut and well defined. In this position the capacity of the tunnel formed by the band and the joint is diminished. Any pathological condition developing in the joint beneath may cause bony, cartilaginous or soft tissue thickening and encroachment on the space necessary for free passage of the ulnar nerve beneath the fibrous band (Fig. 3). Compression of the nerve against the band is then inevitable in the flexed position and a conduction block or neuritis develops.

The compressing action of the transverse band is similar in effect to the transverse carpal ligament at the wrist. Both nerves show superficial flattening at the level of the band and proximal enlargement - the ulnar nerve being thickened in the postcondylar groove behind the medial epicondyle. The demonstration of this simple compression mechanism indicates that 'frictional trauma,' or 'traction' to the nerve, probably does not occur.

In certain cases of neuritis in which some thickening of the ulnar nerve was present, the elbow joint was clinically and radiologically normal. The fibrous band appeared to be constricting the nerve in these cases, and it seemed that the tunnel formed by the band superficially and the joint beneath could reach a critical narrowing when flexion of the elbow would compress the nerve. Several so-called idiopathic cases of ulnar neuritis are due to this cause and are relieved by division of the band. In some individuals simple flexion of the elbow causes 


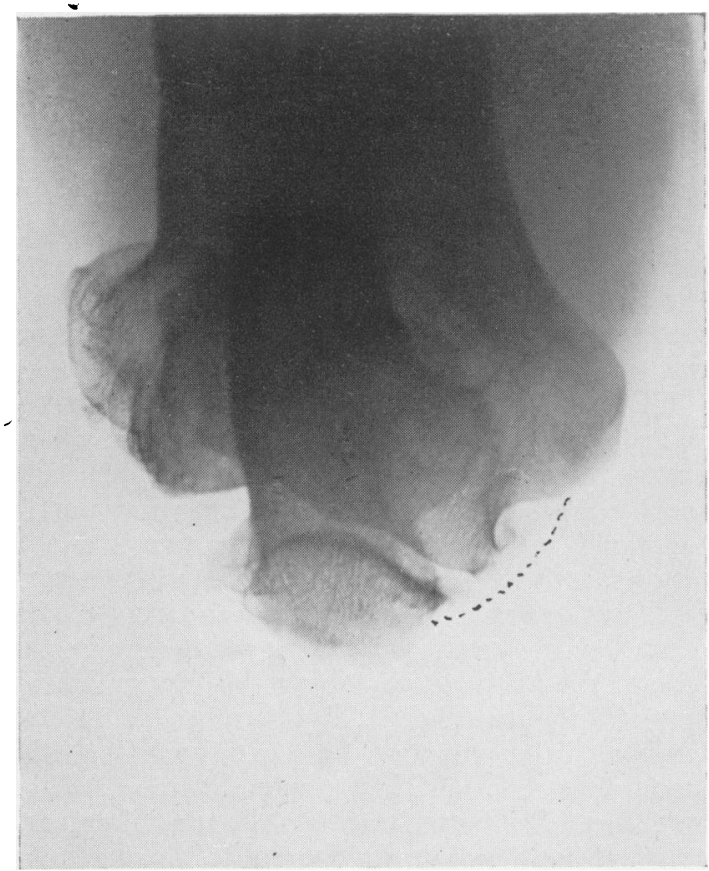

FIG. 3.-Osteoarthritis of elbow joint secondary to old fracture. X-ray taken with elbow in flexion. Large osteophyte arising from edge of trochlea showing obvious encroachment into ulnar nerve groove. Fibrous band indicated by dotted line.

tingling in the little and ring fingers, and temporary neuritis after illness or operations may be due to sleeping with the elbow flexed in the postoperative stage (Osborne, 1958).

3. Certain newer electrical methods of investigating peripheral nerve lesions may be of considerable assistance in differentiating between the varieties of ulnar neuritis. Strength duration curves have a limited use, but may confirm clinical impression of sparing of certain intrinsic muscles. Electromyography may also be of value in following the progress of a doubtful case.

The recording of sensory conduction times (Dawson and Scott, I949; Gilliatt and Sears, 1958), and motor nerve conduction velocity (Simpson, 1956), are elaborate methods of electrical investigation which are of particular value in localizing the exact level of the lesion in the length of the nerve. Sensory impulses are applied to the digital nerves of the little finger. Sensory conduction is slowed through the site of the lesion.

The advantages of these tests are stated by Gilliatt to be firstly, that abnormality may be detected when clinical examination is normal, and secondly, that the test gives an objective result and does not require the co-operation of the patient. Motor nerve conduction tests are carried out by placing an electromyograph recording needle in the muscle, and applying a stimulus to $\mathbb{Q}$ the nerve at varying proximal levels. Conduction $c$. rates can then be studied in different sites of the same nerve.

These electrical methods of investigation require considerable experience and elaborate technical equipment, but may provide information leading to a diagnosis unobtainable in any other way.

\section{Classification or Causes of Ulnar Neuritis At the Level of THE Wrist and HaNd}

I. Compression by ganglia:

(i) Affecting deep motor branch only, causing interosseus palsy.

(ii) Affecting all branches with partial sensory changes and involving hypothenar muscles.

2. Traumatic pressure neuritis due to occupation, e.g. scrubbing or grasping, stiff lever or single injury, e.g. gardening or starting car. In some of these cases the nerve is directly compressed and in others the injury causes a capsular strain from which a carpal ganglion develops.

3. Compression by fibrous band.

At the Level of The Elbow

Compression neuritis of the ulnar nerve can be secondary to:

(i) Arthritis:

Osteoarthritis.

Osteochondritis dissecans with secondary arthritis.

Rheumatoid arthritis.

Tuberculous arthritis.

Neuropathic arthritis secondary to syringomyelia.

(ii) Old fracture:

Malunion of medial epicondylar fracture with deformity of groove.

Supracondylar fracture with malunion and late arthritis.

Cubitus valgus due to old separated capitellar epiphysis - the classical

(iii) Ganglia: ' tardy ulnar palsy.'

Arising from medial capsule of elbow joint or deep in fibres of flexor carpi ulnaris.

(iv) Local injuries to the post-condylar groove:

Causing scarring and constriction of nerve.

(v) Idiopathic:

This group comprises more than 10 per cent. of cases of ulnar neuritis and is due to simple compression by the 
fibrous bridge of flexor carpi ulnaris. Although the elbow joint is normal on physical and radiological examination, some swelling of the ulnar nerve in the post-condylar groove is present in most of these cases.

Clinical Features of Ulnar Neuritis at the Elbow

Gradual compression of the ulnar nerve in the ' cubital' tunnel-a term coined by Fiendel and Stratford (1958)-produces an incomplete nerve lesion in which the interossei muscles of the hand are mainly affected, the hypothenar muscles being less involved and the flexor carpi ulnaris and ulnar part of the flexor profundus digitorum are usually spared. This selective involvement of the hand muscles cannot be explained and creates difficulty in diagnosis, as the picture may be confused with neuritis at wrist level in the absence of a demonstrable ganglion or history of injury.

McGowan (1950) has graded cases according to the severity of nerve involvement into:

Grade I.-Mainly subjective features without motor weakness of hand. In the early stages paraesthesiae and minor hypoaesthesia of the little and ring fingers are present associated with sensations of discomfort and clumsiness in the hand. The nerve is normal in appearance at operation and relief of paraesthesia and sensory symptoms is immediate.

Grade II.-Intermediate lesions with weakness and wasting of interossei but with some residual intrinsic power, hypoaesthesia is present, but is never complete. At operation some swelling of the nerve is present, in the post-condylar groove and narrowing deep to the fibrous arch of the flexor carpi ulnaris.

Grade III.-Severe lesions-marked paralysis of the interossei is present with wasting and weakness of the hypo-thenar muscles and complete or partial anaesthesia. The flexor carpi ulnaris and flexor profundus digitorum muscles may be affected but are never paralysed.

In most cases of ulnar neuritis due to a cause at elbow level the lesion is progressive and, if the compression is unrelieved, permanent intrinsic paralysis of the hand can occur. The primary lesion of the elbow joint in most cases determines the severity of the ulnar neuritis.

Severe progressive lesions-Grades II and III are in most cases due to frank deformity of the elbow joint as a result of old fractures - tardy ulnar palsy, or result from advanced osteoarthritis of the ulnar groove.

Grade I and minor Grade II cases comprise most early lesions, and nearly all the so-called idiopathic cases in which no abnormality of the joint was present and in which the degree of compression of the nerve was obviously not severe.

Features of Ulnar Neuritis at Wrist LeVel

Most of the cases were middle-aged males, and a history of injury, often repeated or occupational, was usually present. If the ulnar palsy is confined to the interossei, sensory symptoms such as pain and parasthesiae may be entirely absent. The ganglion responsible for compression of the deep palmar branch of the nerve is small and usually cannot be clinically identified. Seddon advises operative exploration in all definite cases of this type of neuritis. If repeated occupational trauma seems to be a causative factor-a change of type of work may, however, be all that is necessary and several of the recorded cases have recovered spontaneously (Russell and Whitty, 1947). At operation the ulnar nerve is identified to the level of the hook of the hamate. The superficial sensory branch is retracted and the ganglion, if present,is discovered lying beneath the deep motor branch distal to the branches to the hypothenar muscles. The nerve may be swollen, flattened and tightly stretched over the ganglion which must be removed as completely as possible. Recovery of intrinsic motor power may not occur for six to twelve months after operation, although somes immediate relief is usually obtained.

Ganglia arising more proximally from the wrist may be sometimes identified on physical examination. Partial compression of all the terminal branches of the nerve causes tingling, discomfort and numbness affecting the palmar surfaces of the little and ring fingers and wasting and weakness of the hypothenar and interossei muscles. In the early stages this clinical picture may resemble idiopathic ulnar neuritis occurring at the elbow joint. In this type of case electrical studies, particularly of sensory and motor conduction times, may be helpful in identifying the level of the lesion, but exploration at both wrist and elbow may be sometimes necessary to avoid missing a small ganglion.

Treatment of Ulnar Neuritis at the Elbow ANTERIOR Transposition OF Ulnar Nerve

Since Roux originally performed transposition, this operation had gradually become accepted as the ideal treatment for ulnar neuritis. In most early and intermediate cases the results of this procedure were very satisfactory-full sensory recovery and considerable motor improvement was expected unless paralysis of the intrinsic muscles had occurred. In intermediate cases, recovery to the clinical state existing 12 to 18 months before was usual. The stages of operation were as follows.

I. Eight-inch incision at back of elbow joint. 
The nerve is freed by dividing the fibrous band, and splitting the two heads of origin of flexor carpi ulnaris.

2. The articular branch to the joint can be sacrificed, but, to allow mobilization of the nerve anteriorly, the funiculi supplying the flexor carpi ulnaris muscle must be stripped up in the main trunk for a distance of about $1 \frac{1}{2}$ in.

3. The flexor muscle mass lying anteriorly can be incised deeply to make a bed for the nerve or the nerve can be buried deep to the flexor origin, which is detached and turned down from the epicondyle.

4. It is important to avoid kinking of the nerve: (i) against the medial intermuscular septum, proximally from which a piece should be excised at the site of contact of the nerve; (ii) distally the nerve may become twisted over the fibrous edge of flexor carpi ulnaris which should be divided and opened at this level.

Even with a correct surgical technique, however, the results of transposition are sometimes unpredictable. Residual tenderness and causalgic pain may occur and gross scarring of the muscular bed may cause further damage to the nerve. The operation is not a small procedure, and in the past surgeons have hesitated to explore and transpose the nerve until clinical signs were present and a definite diagnosis was possible. Relief of ulnar neuritis at Stage I should be the aim of treatment, when full recovery could be anticipated. The demonstration of a common compression lesion by Osborne (I957) suggested the simpler operation of decompression of the nerve.

\section{Decompression of Ulnar Nerve}

Performed through a small 3 -in. incision in the line of the nerve centred at the point of the elbow. The swollen nerve is identified and mobilized above the joint and the fibrous band divided as it crosses the nerve transversely. The aponeurosis of the flexor carpi ulnaris is split to expose the flattened segment of nerve which should be gently loosened from its bed. The subcutaneous fat is now sutured over the nerve and the skin closed, the elbow is bandaged lightly in extension for 10 days after which flexion movements are commenced.

The results of this minor procedure in most early cases appear to be as satisfactory as after the more major operation of transposition. The operation has no intrinsic complications and has enlarged the field of surgery to early or mild cases before transposition seems justified. In more severe Grades II and III cases the results also appear to be equally satisfactory, but comparison of these grades is difficult and a detailed analysis is being carried out at present.

\section{Excision of The Medial Epicondyle}

This has recently been suggested by King and Morgan (1959) with gratifying early results and satisfactory late results. The operation is technically simpler and probably less damaging to the nerve than transposition, but I feel that the beneficial effect is probably due to relief of compression and neurolysis and could be achieved by the simple decompression operation described previously.

\section{Conclusions}

I. Ulnar neuritis is due in most cases to a compression lesion of the nerve occurring at wrist or elbow level.

2. The term ' traumatic' is not satisfactory and ' compression neuritis of the ulnar nerve' is more accurately descriptive.

3. The nerve may be compressed by ganglia at wrist or elbow. Continual pressure by grasping or leaning on handles, tools or levers may cause an occupational neuritis, sometimes after a single day's heavy work. Any pathological lesion of the elbow, but usually arthritis or deformity after olœ fractures, can compress the ulnar nerve against the fibrous bridge of the flexor carpi ulnaris.

4. The clinical features of idiopathic compression neuritis at the elbow may be difficult to distinguish from ganglion pressure at the wrist.

5. Simple decompression of the ulnar nerve, by division of the constricting fibrous band on the back of the elbow joint, appears to give in most cases as satisfactory a result as the more elaborate procedure of anterior transposition.

\section{BIBLIOGRAPHY}

BROOKS, D. M. (r952), f. Bone ft Surg., 34b (3), 39 r.

DAWSON, G. D., and SCOTT, J. W. (1949), F. Neurol. Psychiat., I2, 2, 59 .

FIENDEL, W., and STRATFORD, J. (1958), Canad. med. Ass. F., $78,5,35 \mathrm{I}$. GILLIATT, R.W., and SEARS, T. A. (1958), f. Neurol. Psychiat.,
21, rog.

KING, J., and MORGAN, F. P. (1959), F. Bone ft Surg., $4 \mathrm{Ib}$ (1), 5 I McGOWAN, A. (1950), Ibid., 32b (3), 293.

OSBORNE, G. V. (1957), Ibid., 39b (4), 782.

OSBORNE, G. V. (1958), Brit. med. F., January 25.

PANAS (1878), Arch gén. Méd., ii.

PLATT, H. (1926), Brit. F. Surg., 13, 409.

RAMSAY HUNT (191 1), Amer. F. med. Sci., 141, 224.

RUSSELL, W. RI'TCHIE, and WHITTY, C. W. M. (1947), Lancet, i, 828.

SEDDON, H. J. (1952), F. Bone ft Surg., 34b (3), 386.

SIMPSON, J. A. (1956), f. Newrol. Psychiat., 19, 275. 